\title{
NOUVELle
}

\section{Dynamique de la chromatine en réponse aux dommages de I'ADN}

\author{
Une histoire multiéchelle \\ Judith Miné-Hattab ${ }^{1,2 *}$, Xavier Darzacq ${ }^{3}$
}

\author{
${ }^{1}$ Institut Curie, PSL research university, CNRS, UMR3664, \\ 75005 Paris, France. \\ ${ }^{2}$ Sorbonne Université, Institut Curie, CNRS, UMR3664, F-75005 \\ Paris, France. \\ ${ }^{3}$ Division of genetics, genomics and development, Department \\ of molecular and cell biology, University of California, \\ Berkeley, Berkeley, CA 94720 États-Unis. \\ *Auteur correspondant. \\ judith.mine@curie.fr
}

Dynamique de la chromatine en réponse aux cassures double brins L'information génétique contenue à l'intérieur de nos cellules est codée sous forme d'une molécule : I'ADN. Longue d'environ 2 mètres, celle-ci est compactée dans un noyau d'environ $10 \mu \mathrm{m}$ de diamètre, soit 200000 fois plus petit. L'organisation et la dynamique de I'ADN jouent un rôle essentiel dans de nombreux processus biologiques et se trouvent perturbées dans des cellules de tissus malades. Afin de mieux comprendre la dynamique de l'ADN à l'intérieur d'une cellule vivante, en l'occurrence une cellule de bactérie, l'une des approches consiste à suivre, par microscopie de fluorescence le mouvement d'une région spécifique d'ADN appelée «locus ». Pour observer la dynamique d'un locus particulier dans une cellule vivante, il est nécessaire de mettre en place une stratégie d'ingénierie génomique visant à créer un domaine d'ADN visualisable par microscopie. Pour cela, des opérateurs lac0 (ou teto) ${ }^{1}$ sont introduits au locus d'intérêt, auxquels vont se lier leurs partenaires protéiques Lacl (ou TetR) marqués par des fluorophores. Le site d'intérêt est ainsi visible sous la forme d'un point fluorescent en microscopie champ large. En suivant la position de ce point au cours du temps, il est ainsi possible de déterminer la trajectoire suivie par ce locus, et d'en déduire l'espace qu'il explore au sein

${ }^{1}$ L'opéron Lactose (lac0) recrute le répresseur transcriptionnel Lacl. Le locus tet 0 confère la résistance à la tétracycline en absence de son répresseur transcriptionnel TetR.

du noyau. II a ainsi été montré que la chromatine diffuse différemment selon le niveau de différenciation cellulaire, le cycle cellulaire [1], le niveau d'activité transcriptionnelle, etc.

Au cours de la vie d'une cellule, notre génome subit de nombreuses cassures dont les plus génotoxiques sont les cassures double brin [2]. Comment la dynamique de la chromatine est-elle affectée en réponse aux dommages de I'ADN? Dans une étude précédente $[3,4](\rightarrow)$ nous avions suivi le mouvement de deux loci

\section{$(\rightarrow)$ Voir la Nouvelle de J. Miné-Hattab et R. Rothstein, $m / s$ n० 8-9 août-septembre 2012, page 714} génomiques homologues en l'absence et en présence de cassure(s) double-brin dans des cellules diploïdes de levure Saccharomyces cerevisiae. Nous avions alors observé une augmentation importante de la dynamique de la chromatine en réponse à une ou plusieurs cassures double brin. Le site endommagé s'est révélé être le plus mobile, le volume nucléaire exploré par ce locus étant dix fois supérieur en présence de dommage. Cependant, la réponse cellulaire déclenchée par une cassure double brin ne se limite pas au site endommagé. En effet, la dynamique de l'ensemble du génome augmente également, et cela de façon proportionnelle au nombre de cassures double brin induites dans un noyau [3]. À la suite de dommages de I'ADN, la cellule déclenche donc une modification de la dynamique chromatinienne qui se traduit par une hyper-mobilité locale très importante, ainsi que par une hypermobilité globale plus modérée affectant l'ensemble du génome. Les protéines impliquées dans la recombinaison homologue, $\operatorname{Mecl}^{2}, \operatorname{Rad}^{3}[5], \operatorname{Rad} 51^{4}$ et $\mathrm{Sae} 2^{5}$ [3], sont nécessaires pour promouvoir l'hyper-mobilité de la chromatine en réponse aux cassures double brin. L'augmentation de mobilité locale et globale de la chromatine semble favoriser l'appariement entre chromosomes homologues, celui-ci étant dix fois plus fréquent après l'induction d'une cassure double-brin [3]. De nombreuses études (voir la revue [6]), ont également montré que le changement de mobilité observé chez la levure est un mécanisme conservé à travers les organismes [7-9]. Chez les mammifères, l'augmentation de mobilité à la suite de dommages de I'ADN n'est cependant pas observée dans toutes les études, ces variations étant probablement la conséquence du type et du nombre de cassures, de la région chromatinienne endommagée, et de la voie de signalisation utilisée pour réparer ces cassures.

$\varepsilon$ n réponse aux cassures double brin, le coefficient de diffusion de la chromatine reste inchangé $[3,5]$ : le site endommagé explore un espace nucléaire plus large sans pour cela augmenter sa vitesse de déplacement. Afin de mieux comprendre les modifications affectant la chromatine à la suite des dommages

\footnotetext{
${ }^{2}$ Kinase analogue d'ATR, ataxia telangiectasia Rad3-related protein.

${ }^{3} \operatorname{Rad} 9$ partage des similitudes avec Brcal, breast cancer 1 .

${ }^{4}$ La recombinase RAD51 se lie à l'ADN simple-brin, autour duquel elle forme un filament nucléoprotéique.

${ }^{5}$ Sae2 est une endonucléase capable de faire une coupure propre autour d'une lésion double-brin dans I'ADN.
} 
A

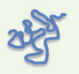

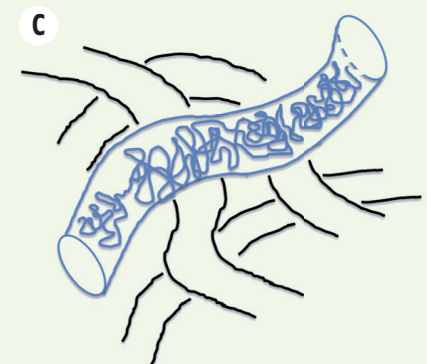

Mouvement du même objet visible à temps long
Mouvement d'un objet Mouvement du même objet visible à temps court visible à temps intermédiaire pendant le temps de l'acquisition, mais le mouvement détaillé de la chromatine pendant cet intervalle de temps reste inconnu. Avec l'arrivée de microscopes plus performants, il est maintenant possible de mesurer la position d'un spot à une vitesse 1000 fois plus importante (toutes les millisecondes). Afin de mettre en évidence l'existence de différents régimes de diffusion, nous avons mesuré la dynamique de la chromatine à 4 échelles de temps différentes, avant et après induction de dommages de I'ADN (une mesure toutes les 10 secondes [3], toutes les secondes, toutes les $100 \mathrm{~ms}$ et même toutes les 10 ms [10]).

de l'ADN, nous avons récemment étudié la dynamique de la chromatine à différentes échelles spatio-temporelles [10].

\section{«Suivi » multi-échelles de la chromatine}

La dynamique de la chromatine peut être mesurée selon différentes échelles de temps variant de quelques secondes à quelques minutes [11]. Lorsque I'on étudie la diffusion d'un objet, l'échelle de temps à laquelle les mesures sont collectées révèle la nature de la diffusion uniquement à cette échelle spatio-temporelle précise. Jusqu'à présent, aucun consensus n'avait été atteint sur la nature exacte du mouvement de la chromatine dans les cellules, sans doute parce que chaque étude se restreignait à une échelle de temps particulière en décrivant un processus de diffusion potentiellement différent à une autre échelle temporelle. De la même façon que la chromatine présente une organisation multi-échelle, on peut imaginer que la chromatine diffuse différemment selon l'échelle spatio-temporelle examinée. Dans ce cas, le mouvement de la chromatine serait décrit par un mouvement complexe résultant de la superposition de différents régimes de diffusion. Par exemple, un locus peut évoluer avec un coefficient de diffusion anormale $A_{\text {micro }}$ dans une région de l'espace qui diffuse elle-même avec un coefficient de diffusion $A_{\text {macro. L'ADN }}$ d'une cellule forme des domaines à plusieurs échelles imbriqués les uns dans les autres, et il est donc concevable qu'un grand domaine, lui-même mobile, contienne un sous-domaine qui en plus de la mobilité qui lui est imposée par le grand domaine, diffuse au sein de ce dernier et ainsi se déplace à plusieurs échelles temporelles et spatiales. Un mouvement complexe peut également résulter de l'alternance d'une diffusion libre et d'une diffusion dirigée, par exemple si l'objet étudié s'accroche par intermittence à un filament d'actine ou de microtubules. La Figure 1, inspirée du modèle de reptation développé par Pierre-Gilles de Gennes (1932-2007)6 [12], illustre un mouvement complexe qui présente différents régimes de diffusion selon l'échelle temporelle examinée.

Jusqu'à présent, la dynamique de la chromatine a été étudiée principalement à des échelles de temps relativement grandes : par exemple, la position d'un site lacO-Lacl est mesurée toutes les 10 secondes. Cet intervalle de temps correspond au temps nécessaire au microscope pour mesurer la position d'un spot en 3 dimensions; la position du spot est alors moyennée

\footnotetext{
${ }^{6}$ Concept de «reptation » décrit par P.-G. de Gennes dans lequel une molécule se déplace tel un serpent en utilisant des supports, filaments d'actine ou microtubules.
}

\section{Le suivi multi-échelle révèle le} mouvement complexe de la chromatine en réponse aux dommages de I'ADN

En suivant le mouvement de la chromatine à différentes échelles temporelles, nous avons montré que la dynamique de la chromatine est plus complexe que nous ne l'anticipions [10]. À la suite de dommages de l'ADN, la chromatine est plus mobile lorsqu'elle est observée à des intervalles de temps de l'ordre de la seconde. Cependant, de manière surprenante, elle est moins mobile lorsqu'elle est examinée à des intervalles de temps très courts (une mesure au maximum toutes les 100 millisecondes). Cela se traduit par un croisement des courbes de déplacements carrés moyens (mean square displacement ou MSD) traduisant le mode de diffusion d'un locus (Figure 2A). En d'autres termes, l'augmentation de mobilité décrite précédemment dans de nombreuses études $[3,5]$ se révèle n'être en fait que la partie apparente, visible à des temps longs, des modifications affectant la chromatine après des dommages de I'ADN. De manière intéressante, l'augmentation de mobilité à des temps longs ainsi que la réduction de mobilité à des temps courts sont toutes deux observées à la fois au site de cassure et dans l'ensemble du génome. Les changements de mobilité de la chromatine observés aux différentes échelles de temps sont donc 


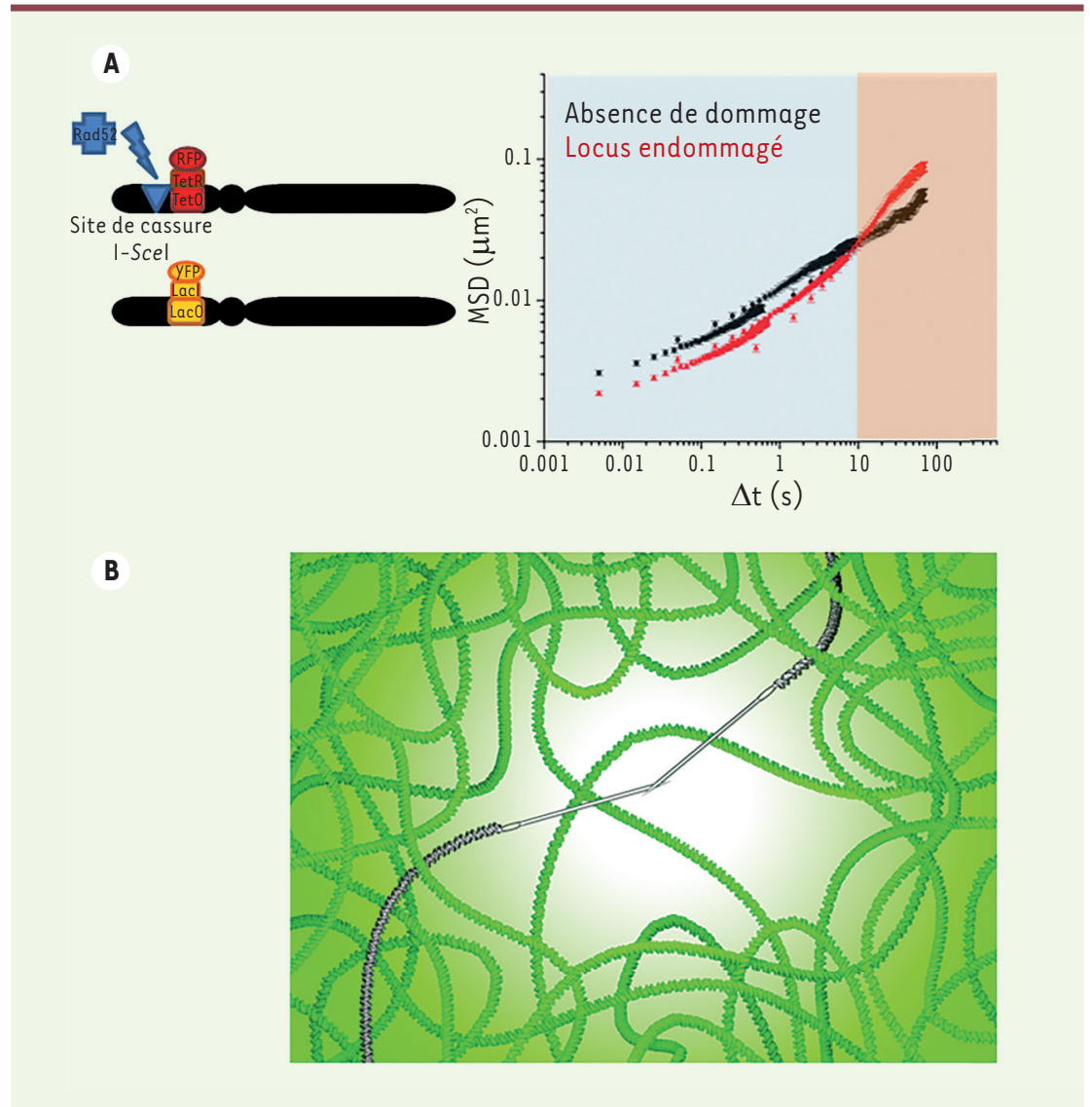

Figure 2. Analyse de la mobilité de la chromatine à différentes échelles de temps. A. À gauche : schéma de la souche utilisée pour décrire la dynamique de la chromatine au locus où la cassure est provoquée. Des opérateurs lacO (ou tetO) sont introduits au locus d'intérêt, auxquels vont se lier leurs partenaires de liaison Lacl (ou TetR) marqués par des fluorophores. RFP (red fluorescent protein) et YFP (yellow fluorescent protein) sont des protéines naturellement fluorescentes, respectivement rouge et jaune. Les protéines impliquées dans la recombinaison homologue, dont Rad51, sont recrutées au site de cassure de l'ADN, le site de reconnaissance de l'endonucléase I-Scel. À droite : carrés moyens des déplacements (MSD) représentés sur une échelle log-log, en l'absence de cassure (courbe noire) et en présence d'une cassure unique au site de cassure de l'endonucléase I-Scel (courbe rouge). B. Modèle de la pelote de laine et de l'aiguille proposé pour illustrer la dynamique multi-échelle de la chromatine en réponse aux dommages de l’ADN.

à la fois locaux et globaux [10]. La protéine Rad51, qui joue un rôle clé dans le processus de recombinaison homologue, est essentielle pour les changements de mobilité observés à toutes les échelles de temps.

Dynamique multi-échelle et lien avec la rigidité de la chromatine Afin d'interpréter nos résultats expérimentaux, nous avons comparé les profils de MSD obtenus (traduisant la mobilité du locus observé) (Figure 2A) du locus endommagé que dans le reste du génome, nous avons proposé que l'augmentation de rigidité devait être plus importante à ce niveau, même si elle affecte également l'ensemble du génome dans une moindre mesure. Nos résultats sont concordants avec une étude récemment réalisée avec des méthodes différentes sur des cellules de levure haploïdes [14].

Nous avons proposé un modèle dans lequel la chromatine peut être comparée à une pelote de laine: en réponse aux dommages de l'ADN, les extrémités endommagées sont recouvertes de protéines de recombinaison homologue, symbolisées dans ce modèle par une aiguille (Rad51 étant connu pour rigidifier l'ADN in vitro). En réponse aux dommages, la chromatine deviendrait globalement plus rigide, cet effet étant plus important au niveau de la cassure. À intervalles de temps courts, l'aiguille est peu mobile car très rigide ; au contraire, à intervalles de temps longs, l'aiguille pourrait traverser les mailles de laine plus facilement et deviendrait plus mobile qu'avant la cassure de l'ADN. De façon générale, une dynamique multi-échelle est-t-elle un mécanisme favorisant la cinétique de recherche de cibles? Dans de futurs travaux, il serait intéressant d'examiner si d'autres mécanismes biologiques font intervenir de tels mouvements résultant de la superposition de différents types de diffusion. $\diamond$

Chromatin mobility upon DNA damage: a multi-scale story

\section{LIENS D'INTÉRÊT}

Les auteurs déclarent n'avoir aucun lien d'intérêt concernant les données pubiées dans cet article.

\section{RéFÉRENCES}

1. Heun P, Laroche T, Shimada K, et al. Chromosome dynamics in the yeast interphase nucleus. Science 2001 ; 294 : 2181-6.

2. Sung P. Introduction to the thematic minireview series: DNA double-strand break repair and pathway choice. J Biol Chem 2018; doi: 10.1074/jbc. TM118.003212.

3. Miné-Hattab J, Rothstein R. Increased chromosome mobility facilitates homology search during recombination. Nat Cell Biol $2012 ; 14$ : 510-7. 


\section{RéFÉRENCES}

4. Miné-Hattab J, Rothstein R. Réparation de I'ADN Comment trouver le bon partenaire? Med Sci (Paris) $2012 ; 28: 714-6$.

5. Dion V, Kalck V, Horigome C, et al. Increased mobility of double-strand breaks requires $\mathrm{Mecl}$, $\operatorname{Rad} 9$ and the homologous recombination machinery. Nat Cell Biol $2012 ; 14: 502-9$.

6. Miné-Hattab J, Rothstein R. DNA in motion during double-strand break repair. Trends Cell Biol 2013;23 529-36.

7. Aten JA, Stap J, Krawczyk PM, et al. Dynamics of DNA double-strand breaks revealed by clustering of damaged chromosome domains. Science 2004 ; 303 : 92-5.

8. Chiolo I, Minoda A, Colmenares SU, et al. Doublestrand breaks in heterochromatin move outside of a dynamic HPla domain to complete recombinational repair. Cell $2011 ; 144: 732-44$.

9. Krawczyk PM, Borovski T, Stap J, et al. Chromatin mobility is increased at sites of DNA double-strand breaks. J Cell Sci 2012 ; $125: 2127-33$.

10. Miné-Hattab J, Recamier V, Izeddin I, et al. Multi-scale tracking reveals scale-dependent chromatin dynamics after DNA damage. Mol Biol Cell 2017 ; doi: 10.1091/mbc.E17-05-0317.
11. Hajjoul H, Mathon J, Ranchon H, et al, Highthroughput chromatin motion tracking in living yeast reveals the flexibility of the fiber throughout the genome. Genome Res $2013 ; 23$ : 1829-38.

12. De Gennes PG. Kinetics of diffusioncontrolled processes in dense polymer systems. II.Effects of entanglements. J Chem Phys 1982 ; 76 : 3322-6.

13. Faller R, Müller-Plathe F. Chain stiffness intensifies the reptation characteristics of polymer dynamics in the melt. Chemphyschem $2008 ; 2: 180-4$.

14. Herbert S, Brion A, Arbona JM, et al. Chromatin stiffening underlies enhanced locus mobility after DNA damage in budding yeast. EMBO J 2017 ; 36 : 2595-608.

\section{NOUVELLE}

\section{La proximité de lésions dans I'ADN favorise la mutagenèse}

Luisa Laureti, Vincent Pagès
CRCM, Centre de recherche en cancérologie de Marseille, CNRS, Inserm, Université Aix Marseille, Institut Paoli-Calmettes,

27, boulevard Lei Roure, 13009 Marseille, France.

vincent.pages@inserm.fr

d'ADN simple-brin générée en aval de la lésion envahit la région homologue (double-brin) de la chromatide sœur ce qui permet à la cellule de récupérer l'information génétique altérée par la lésion. Ce mécanisme contrairement à la TLS est fidèle.

L'équilibre entre ces deux voies de tolérance des dommages est très important puisqu'il définit le niveau de mutagenèse de la cellule. Par conséquent, décrypter la réplication d'un ADN endommagé, en particulier l'équilibre entre synthèse translésionnelle et mécanismes de contournement des dommages, est fondamental pour comprendre comment les mutations apparaissent et comment elles sont évitées. Si d'un côté cette mutagenèse est essentielle au processus d'évolution et d'adaptation au stress de l'environnement, en particulier chez les microorganismes, elle est également responsable de graves dérèglements cellulaires qui peuvent notamment conduire à la cancérisation des cellules humaines. À l'heure actuelle, on ne sait pas comment une cellule dont I'ADN est lésé va choisir une voie de réparation plutôt que l'autre. 